11. Ziegler, S.F. 2006. FOXP3: of mice and men. Annu. Rev. Immunol. 24:209-226.

12. Allan, S.E., et al. 2005. The role of 2 FOXP3 isoforms in the generation of human $\mathrm{CD}^{+}$ Tregs. J. Clin. Invest. 115:3276-3284. doi:10.1172/ JCI24685

13. Walker, M.R., et al. 2003. Induction of FoxP3 and acquisition of $\mathrm{T}$ regulatory activity by stimulated human $\mathrm{CD}^{+} \mathrm{CD} 25^{-} \mathrm{T}$ cells. J. Clin. Invest. 112:1437-1443. doi:10.1172/JCI200319441.

14. Thornton, A.M., and Shevach, E.M. 1998. CD4+CD25+ immunoregulatory $\mathrm{T}$ cells suppress polyclonal $\mathrm{T}$ cell activation in vitro by inhibiting interleukin 2 production. J. Exp. Med. 188:287-296.

15. Von Boehmer, H. 2005. Mechanisms of suppression by suppressor T cells. Nat. Immunol. 6:338-344.

16. Brunkow, M.E., et al. 2001. Disruption of a new forkhead/winged-helix protein, scurfin, results in the fatal lymphoproliferative disorder of the scurfy mouse. Nat. Genet. 27:68-73.

17. Lyon, M.F., Peters, J., Glenister, P.H., Ball, S., and Wright, E. 1990. The scurfy mouse mutant has previously unrecognized hematological abnormalities and resembles Wiskott-Aldrich syndrome. Proc. Natl. Acad. Sci. U. S. A. 87:2433-2437.

18. Clark, L.B., et al. 1999. Cellular and molecular characterization of the scurfy mouse mutant. J. Immunol. 162:2546-2554.

19. Wildin, R.S., et al. 2001. X-linked neonatal diabetes mellitus, enteropathy and endocrinopathy syndrome is the human equivalent of mouse scurfy. Nat. Genet. 27:18-20.

20. Bennett, C.L., et al. 2001. The immune dysregulation, polyendocrinopathy, enteropathy, X-linked syndrome (IPEX) is caused by mutations of FOXP3. Nat. Genet. 27:20-21.

21. Chatila, T.A., et al. 2000. JM2, encoding a fork head-related protein, is mutated in X-linked autoimmunity-allergic disregulation syndrome. J. Clin. Invest. 106:R75-R81.

22. Bacchetta, R., et al. 2006. Defective regulatory and effector $\mathrm{T}$ cell functions in patients with FOXP3 mutations. J. Clin. Invest. 116:1713-1722. doi:10.1172/JCI25112.

23. Lin, W., et al. 2005. Allergic dysregulation and hyperimmunoglobulinemia $\mathrm{E}$ in Foxp3 mutant mice. J. Allergy Clin. Immunol. 116:1106-1115.

24. Schubert, L.A., Jeffery, E., Zhang, Y., Ramsdell, F., and Ziegler, S.F. 2001. Scurfin (FOXP3) acts as a repressor of transcription and regulates $\mathrm{T}$ cell activation. J. Biol. Chem. 276:37672-37679.

\title{
Of mice, men, and elephants: Mycobacterium tuberculosis cell envelope lipids and pathogenesis
}

\author{
Lee W. Riley
}

\author{
Division of Infectious Diseases, School of Public Health, University of California, Berkeley, California, USA.
}

\begin{abstract}
Mycolic acids and structures attached to them constitute a major part of the protective envelope of Mycobacterium tuberculosis, and for this reason, their role in tuberculosis pathogenesis has been extensively studied. In this issue of the JCI, Rao et al. examine the effect of trans-cyclopropanation of oxygenated mycolic acids attached to trehalose dimycolate (TDM) on the murine immune response to infection (see the related article beginning on page 1660). Surprisingly, they found that an M. tuberculosis mutant lacking trans-cyclopropane rings was hypervirulent in mice. The recent recognition of a hypervirulence phenotype in mice associated with laboratory and clinical M. tuberculosis strains with altered cell wall components has provided new insights into how M. tuberculosis may establish persistent infection. However, to date, characterization of these bioactive products in pathogenesis has been largely reductionistic; the relationship of their effects observed in mice to the persistent infection and tuberculosis caused by M. tuberculosis observed in humans remains obscure.
\end{abstract}

The report by Rao et al. in this issue of the JCI (1) represents the latest chapter in a long history of studies examining the role of Mycobacterium tuberculosis bioactive cell envelope lipids in tuberculosis pathogenesis. To be sure, this history goes back more than 50 years, when Middlebrook et al. redescribed a characteristic broth-growth morphology (first described by Robert Koch in 1884) called cording associated with virulent tubercle bacilli (2). Subsequently, Hubert Bloch characterized the effect of petroleum ether-extractable "cord factor"

Nonstandard abbreviations used: $c m a A 2$, cyclopropane mycolic acid synthase 2; PGL, phenolic glycolipid; TDM, trehalose dimycolate.

Conflict of interest: The author has declared that no conflict of interest exists.

Citation for this article: J. Clin. Invest. 116:1475-1478 (2006). doi:10.1172/JCI28734. from $M$. tuberculosis on leukocyte migration and toxicity in mice (3). The toxicity in mice was demonstrated only after serial injections of the lipid extract, suggesting that the disease involved cell-mediated immunopathology (3). Ultimately, these effects of cord factor were traced to the glycolipid trehalose dimycolate (TDM) $(4,5)$. It later turned out that TDM is found in pathogenic as well as saprophytic mycobacteria (6). Recent studies suggest, however, that the relative composition and structural differences in TDM among different mycobacteria influence the host animal biologic response.

\section{Mycolic acids and their effect on host immune response}

TDM is composed of a nonreducing sugar trehalose covalently linked to mycolic acids.
Mycolic acids are $\beta$-hydroxyl fatty acids with an $\alpha$-alkyl side chain (7) (Figure 1). The M. tuberculosis cell wall contains 3 classes of mycolic acids: $\alpha$-, keto-, and methoxymycolates. $\alpha$-Mycolic acid has 2 cyclopropane rings, which are in the cis configuration (8, 9), while the keto- and methoxymycolates have 1 ring each in either cis or trans configuration (10). In addition to trehalose, mycolates are linked to arabinogalactan, which is covalently attached to the peptidoglycan layer (Figure 1) (11).

The relative composition of oxygenated mycolates influences the intramacrophage growth rate of $M$. tuberculosis (12). Strains lacking ketomycolates become reduced in their ability to grow inside THP-1 cells (12). Dubnau et al. reported that the absence of keto- and methoxymycolates leads to attenuation of $M$. tuberculosis in mice (13). Wild-type M. bovis strains possess a complete set of mycolic acids, but the vaccine strain $M$. bovis BCG-Pasteur lacks methoxymycolates (13). These observations indicate that differences in the relative composition of mycolates can influence the host immune response.

The report by Rao et al. goes further and shows that alteration in the trans-cyclopropane rings of mycolic acids in TDM affects the mouse immune response (1). They showed that a cyclopropane mycolic acid synthase 2 ( $c m a A 2)$ mutant of M. tuberculosis, which lacks trans-cyclopropane rings, is hypervirulent in mice. Accelerated mortality in mice was attributed to severe lung lesions 


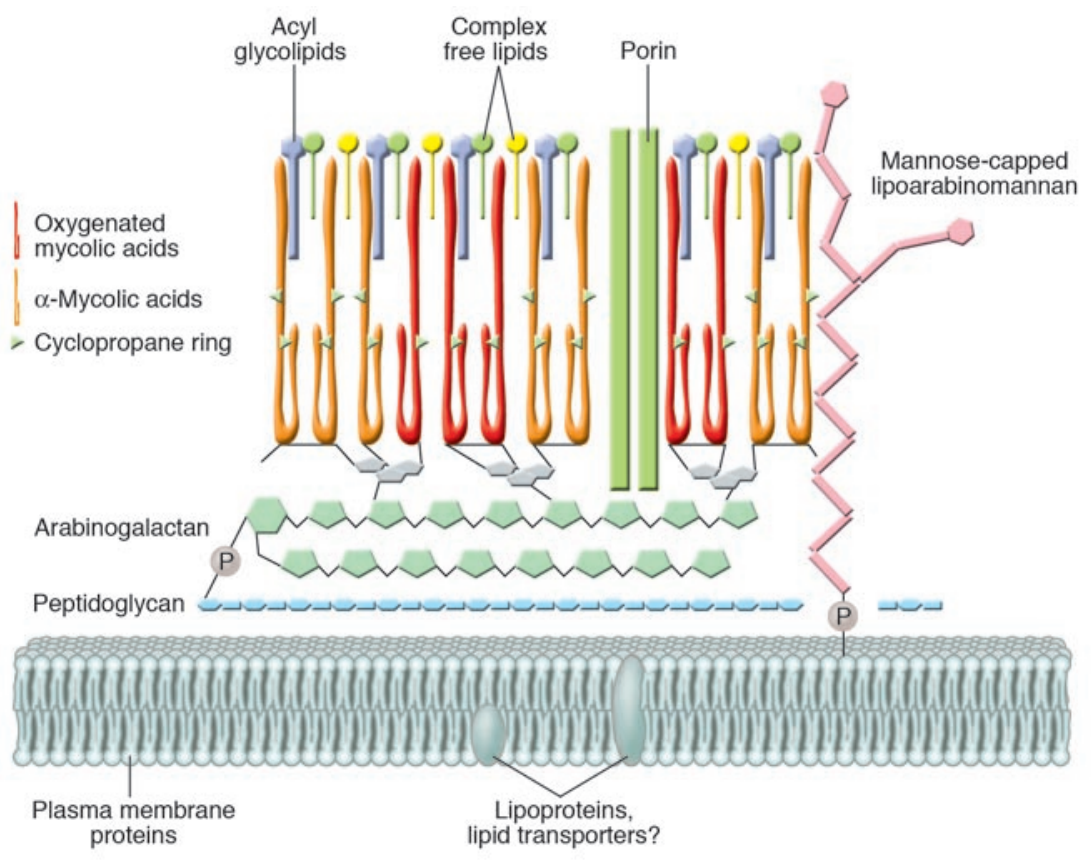

Figure 1

Schematic representation of $M$. tuberculosis cell envelope. Three forms of mycolic acids are depicted. $\alpha$-Mycolates are the most abundant form in M. tuberculosis (orange). It has 2 cyclopropane rings (triangles) in cis configuration. Oxygenated mycolates (keto- and methoxy-, shown in red) have one cyclopropane ring each that is in either cis or trans configuration. They are covalently linked to the arabinogalactan layer, which is linked to the peptidoglycan layer. Other lipid complexes in the cell wall include acyl glycolipids (including TDM) and other complex free lipids (e.g., phthiocerol dimycocerosate) as well as sulfolipids. Lipoarabinomannan is shown linked to the plasma membrane via a phosphodiester bond. Rao et al. (1) found that the oxygenated mycolates of a cmaA2 M. tuberculosis mutant lack trans-cyclopropanated rings. Such a change could affect the mycolate layer of the cell envelope, which they claim causes a hyperinflammatory response in mice. Figure adapted and modified with permission from the Annual Review of Biochemistry (11) and Cellular Microbiology (29). resulting from the excessive inflammatory response produced by the $\mathrm{cmaA} 2$ mutant. In a clever experiment using surface lipid extracts of the mutant and wild-type strains, they showed that macrophages infected with the delipidated wild-type strain reconstituted with the lipid extract of the mutant showed a 2-fold higher expression of TNF- $\alpha$ than did macrophages infected with the intact wild-type strain. The delipidated mutant reconstituted with the wildtype lipid extract exhibited the wild-type phenotype. Thus, the hyperinflammatory response could be specifically attributed to the TDM lacking trans-cyclopropane rings.

The same group previously described another cyclopropane synthase gene $-p c a A$ - that is needed for synthesis of the proximal cyclopropane ring of $\alpha$-mycolic acid as well as cord formation $(14,15)$. The $M$. tuberculosis pcaA mutant was attenuated in mice, and TDM purified from it was hypoinflammatory (15). Thus, trans-cyclopropanation of methoxy- and ketomycolic acids in TDM and cis-cyclopropanation of $\alpha$-mycolates in M. tuberculosis appear to elicit opposite effects on the immune response of mice. These findings support the idea that fine structural differences in TDM do influence clinical outcome, at least in the mouse model.

Modulation of the host immune response by $M$. tuberculosis cell envelope lipids is not limited to mycolated products. Other products shown to influence host innate and adaptive immune responses include lipoglycans such as lipoarabinomannan and lipomannan (16-18), a 19-kDa lipoprotein that stimulates TLR2 (19), sulfolipids (20), and the cell wall-associated complex free lipid phthiocerol dimycocerosate (21-23).

\section{The relationship between bioactive lipids and the disease process}

New genetic techniques applied to mycobacteria, as well as mycobacterial genomics and advances in mass spectrometry of lipids, have helped to describe the biologic effects of these lipid-associated products in the host. However, many overlapping and sometimes contradictory observations have also obfuscated our understanding of the relationship of these lipid products to M. tuberculosis's ability to establish infection and cause tuberculosis. It is not surprising that these cell wall products have the observed effects on host immunity. What is not known is how changes in specific lipid structures or composition modify the whole bacterial cell wall architecture, which in turn may affect how the bacillus influences infection outcome in a host. In many ways, approaches used to examine bacterial products in isolation to infer the pathogenic strategies of $M$. tuberculosis are reminiscent of the proverbial 6 blind men trying to describe an elephant (see "The Blind Men and the Elephant"). The relevance of these observations to human infection spontaneous clearance, persistent infection, rapidly progressive disease, and reactivation disease - remains obscure.

In humans, $M$. tuberculosis elicits a variety of clinical outcomes, even among those individuals with no apparent host-related predisposing conditions. While M. tuberculosis strains characteristically show little genetic heterogeneity, recent epidemiologic studies suggest that some $M$. tuberculosis strains have an enhanced ability to modify the host cellular immune response (24-27). Such strains are over-represented in certain communities. For example, strain CDC1551 caused a large outbreak of tuberculosis in rural counties in Tennessee and Kentucky in 1994-1996 (24). In mice, it was found to produce an enhanced proinflammatory cytokine response (25). This response was attributed to the apolar lipids of this strain (25). Another strain (HN878), a member of a clade belonging to the Beijing family, caused a prison outbreak in Texas (26). HN878 was found to express a highly biologically active lipid species, phenolic glycolipid (PGL), not detected in M. tuberculosis strain CDC1551 or the laboratory strain $\mathrm{H} 37 \mathrm{Rv}$ (27). In the mouse model, this strain exhibited "hyperlethality." Disruption of the HN878 polyketide synthase gene cluster pks1-pks15 led to loss of PGL, which was associated with increased release of TNF- $\alpha$, IL- 6 , and IL- 12 by macrophages infected with the mutant in vitro. The investigators further showed that PGL itself had an inhibitory effect on proinflammatory cytokine release by macrophages.

The above examples illustrate the relevance of differing lipid profiles to human disease. Large outbreaks were caused by both CDC1551 and HN878, indicating that 


\section{The blind men and the elephant}

It was six men of Indostan

To learning much inclined,

Who went to see the Elephant

(Though all of them were blind),

That each by observation

Might satisfy his mind.

The First approached the Elephant,

And happening to fall

Against his broad and sturdy side,

At once began to bawl:

"God bless me! but the Elephant

Is very like a wall!"

The Second, feeling of the tusk,

Cried: "Ho! what have we here

So very round and smooth and sharp?

To me 'tis mighty clear

This wonder of an Elephant

Is very like a spear!"
The Third approached the animal,

And, happening to take

The squirming trunk within his hands,

Thus boldly up and spake:

"I see," quoth he, "the Elephant

Is very like a snake!"

The Fourth reached out his eager hand, And felt about the knee.

"What most this wondrous beast is like

Is mighty plain," quoth he;

"Tis clear enough the Elephant

Is very like a tree!"

The Fifth, who chanced to touch the ear, Said: "E'en the blindest man

Can tell what this resembles most;

Deny the fact who can,

This marvel of an Elephant

Is very like a fan!"
The Sixth no sooner had begun

About the beast to grope,

Than, seizing on the swinging tail

That fell within his scope,

"I see," quoth he, "the Elephant

Is very like a rope!"

And so these men of Indostan

Disputed loud and long,

Each in his own opinion

Exceeding stiff and strong,

Though each was partly in the right

And all were in the wrong!

\section{Moral}

So oft in theologic wars,

The disputants, I ween,

Rail on in utter ignorance,

Of what each other mean,

And prate about an Elephant

Not one of them has seen!

American poet John Godfrey Saxe (1816-1887), based on an Indian fable

these strains had distinct properties that made them highly successful in transmitting and causing disease in humans. Yet the distinct biologic properties that were identified - lipid products - and their effect in an animal model showed them to elicit opposite responses. Wild-type CDC1551 elicited a strong Th1 type response, while wild-type HN878 induced a diminished Th1 type response and was hypervirulent in mice. These distinct properties do not appear to explain the strains' success in the human population.

\section{Spectrum of host immune response elicited by $M$. tuberculosis cell envelope alteration}

The above, seemingly contradictory observations actually serve to provide new insights about tuberculosis pathogenesis. Recently, M. tuberculosis H37Rv and Erdman strains disrupted in a 13-gene operon called mce1 were shown to become hypervirulent in mice (28). The mce1 operon encodes components of an $\mathrm{ABC}$ transporter, including a putative lipoprotein, that are located in the cell wall. Like HN878, the mce1 operon mutants are unable to elicit a strong Th1 type immune response; they cause aberrant migration of proinflammatory cells and are unable to elicit organized granuloma formation in mouse lungs. Thus, as is reported here by Rao et al. (1), laboratory-constructed strains of M. tuberculosis, with modified cell walls, can mimic the effect of different clinical isolates on immunocompetent mice.

These studies with human clinical and laboratory strains of $M$. tuberculosis show that changes in the cell wall can elicit a wide spectrum of immune responses in mice that range from a hyperinflammatory response (as in the case of the cmaA2 mutant), which can cause death, to a hypoinflammatory response (as in the case of HN878 and the mce1 operon mutants), which can also cause death in mice. For M. tuberculosis to persist inside a host, an intact granuloma comprised of just enough proinflammatory cells to not be detrimental to the bacilli appears to be necessary.

It should be pointed out that cells that comprise granulomas are mortal and that they constantly undergo replacement. Necrosis, a hallmark of tuberculosis disease, results when the programmed cell death rate in the granuloma exceeds cell replacement, which triggers uncontrolled bacterial proliferation. On the other hand, if the rate of new proinflammatory cell migration to the site of granulomas exceeds cell death rate, a hyperinflammatory state is established. In either case, these 2 extreme responses are detrimental to the host and, ultimately, to the bacilli. The variety of host immune responses elicited by $M$. tuberculosis lipids suggests that in a natural infection $M$. tuberculosis can continually remodel its cell wall, perhaps in response to granuloma cell turnover, in order to maintain the equilibrium of proinflammatory cells that make the granuloma permissive for both the bacilli and the host.

The story of the role of $M$. tuberculosis bioactive cell envelope lipids in host infection began many years ago with a chapter describing the tail, or cord, of $M$. tuberculosis. Rao et al. (1) have added a new chapter to this story by describing the importance of the shape of this tail. Hopefully, the next chapter can begin to describe the whole elephant.

\section{Acknowledgments}

I thank Nicola Casali and Sally Cantrell for critical review of this manuscript.

Address correspondence to: Lee W. Riley, Division of Infectious Diseases, School of Public Health, University of California, 223 Warren Hall MC 7360, Berkeley, California 94720-7360, USA. Phone: (510) 642-9200; Fax: (510) 642-6350; E-mail: lwriley@berkeley.edu.

1. Rao, V., Gao, F., Chen, B., Jacobs, W.R., Jr., and Glickman, M.S. 2006. Trans-cyclopropanation of mycolic acids on trehalose dimycolate suppresses Mycobacterium tuberculosis-induced inflammation and virulence. J. Clin. Invest. 116:1660-1667. doi:10.1172/JCI27335.

2. Middlebrook, G., Dubos, R, and Pierce, C. 1947. Virulence and morphological characteristics of mammalian tubercle bacilli. J. Exp. Med. 86:175-184.

3. Bloch, H. 1950. Studies on the virulence of tubercle bacilli. Isolation and biological properties of a constitutent of virulent organisms. J. Exp. Med. 91:197-218. 4. Noll, H., and Bloch, H. 1955. Studies on the chem- 
istry of the cord factor of Mycobacterium tuberculosis. J. Biol. Chem. 214:251-265.

5. Noll, H., Asselineau, J., and Lederer, E. 1956. The chemical structure of the cord factor of Mycobacterium tuberculosis. Biochim. Biophys. Acta. 20:299-309.

6. Goren, M.B. 1972. Mycobacterial lipids: selected topics. Bacteriol. Rev. 36:33-64.

7. Asselineau, J., and Lederer, E. 1950. Structure of the mycolic acids of mycobacteria. Nature. 166:782-783.

8. George, K.M., Yuan, Y., Sherman, D.R., and Barry, C.E., 3rd. 1995. The biosynthesis of cyclopropanated mycolic acids in Mycobacterium tuberculosis. Identification and functional analysis of CMAS- 2 . J. Biol. Chem. 270:2792-2798.

9. Yuan, Y., Lee, R.E., Besra, G.S., Belisle, J.T., and Barry, C.E., 3rd. 1995. Identification of a gene involved in the biosynthesis of cyclopropanated mycolic acids in Mycobacterium tuberculosis. Proc. Natl. Acad. Sci. U. S. A. 92:6630-6634.

10. Barry, C.E., 3rd, et al. 1998. Mycolic acids: structure, biosynthesis, and physiological function. Prog. Lipid Res. 37:143-179.

11. Brennan, P.J., and Nikaido, H. 1995. The envelope of mycobacteria. Annu. Rev. Biochem. 64:29-63.

12. Yuan, Y., YaQi, Z., Crane, D.D., and Barry, C.E., 3rd. 1998. The effect of oxygenated mycolic acid composition on cell wall function and macrophage growth in Mycobacterium tuberculosis. Mol. Microbiol. 29:1449-1458.

13. Dubnau, E., et al. 2000. Oxygenated mycolic acids are necessary for virulence of Mycobacterium tubercu- losis in mice. Mol. Microbiol. 36:630-637.

14. Glickman, M.S., Cox, J.S., and Jacobs, W.R., Jr. 2000 A novel mycolic acid cyclopropane synthetase is required for cording, persistence, and virulence of Mycobacterium tuberculosis. Mol. Cell. 5:717-727.

15. Rao, V., Fujiwara, N., Porcelli, S.A., and Glickman, M.S. 2005. Mycobacterium tuberculosis controls host innate immune activation through cyclopropane modification of a glycolipid effector molecule. J. Exp. Med. 201:535-543.

16. Chatterjee D., and Khoo, K.H. 1998. Mycobacterial lipoarabinomannan: an extraordinary lipoheteroglycan with profound physiological effects. Glycobiology. 8:113-120.

17. Chatterjee D., Roberts, A.D., Lowell, K., Brennan, P. and Orme, I.M. 1992. Structural basis of capacity of lipoarabinomannan to induce secretion of tumor necrosis factor. Infect. Immun. 60:1249-1253.

18. Quesniaux, V.J., et al. 2004. Toll-like receptor 2 (TLR2)-dependent-positive and TLR2-independent-negative regulation of proinflammatory cytokines by mycobacterial lipomannans. J. Immu nol. 172:4425-4434.

19. Brightbill, H.D., et al. 1999. Host defense mechanisms triggered by microbial lipoproteins through toll-like receptors. Science. 285:732-736.

20. Goren, M.B., Broki, O., and Das, B.C. 1976. Sulfatides of Mycobacterium tuberculosis: the structure of the principal sufatide (SL-1). Biochemistry. 15:2728-2735.

21. Camacho, L.R., et al. 2001. Analysis of the phthiocerol dimycocerosate locus of Mycobacterium tuberculo- sis. Evidence that this lipid is involved in the cell wall permeability barrier. J. Biol. Chem. 276:19845-19854.

22. Cox, J.S., Chen, B., McNeil, M., and Jacobs, W.R., Jr. 1999. Complex lipid determines tissue-specific replication of Mycobacterium tuberculosis in mice. Nature. 402:79-83.

23. Rousseau, C., et al. 2003. Virulence attenuation of two Mas-like polyketide synthase mutants of Mycobacterium tuberculosis. Microbiology. 149:1837-1847.

24. Valway, S.E., et al. 1998. An outbreak involving extensive transmission of a virulent strain of Mycobacterium tuberculosis. N. Engl. J. Med. 338:633-639.

25. Manca, C., et al. 1999. Mycobacterium tuberculosis CDC1551 induces a more vigorous host response in vivo and in vitro, but is not more virulent than other clinical isolates. J. Immunol. 162:6740-6746.

26. Sreevatsan, S., et al. 1997. Restricted structural gene polymorphism in the Mycobacterium tuberculosis complex indicates evolutionarily recent global dissemination. Proc. Natl. Acad. Sci. U. S. A. 94:9869-9874

27. Reed, M.B., et al. 2004. A glycolipid of hypervirulent tuberculosis strains that inhibits the innate immune response. Nature. 431:84-87.

28. Shimono, N., et al. 2003. Hypervirulent mutant of Mycobacterium tuberculosis resulting from disruption of the mce1 operon. Proc. Natl. Acad. Sci. U. S. A. 100:15918-15923.

29. Karakousis, P.C., Bishai, W.R., and Dorman, S.E. 2004. Mycobacterium tuberculosis cell envelope lipids and the host immune response. Cell. Microbiol. 6:105-116.

\title{
Probing the role of stearoyl-CoA desaturase-1 in hepatic insulin resistance
}

\author{
Matthew T. Flowers, ${ }^{1}$ Makoto Miyazaki, ${ }^{2}$ Xueqing Liu, ${ }^{2}$ and James M. Ntambi1,2 \\ 1Department of Nutritional Sciences and 2Department of Biochemistry, University of Wisconsin — Madison, Madison, Wisconsin, USA.
}

Previous studies using stearoyl-CoA desaturase-1-deficient (SCD1-deficient) mice have shown that this enzyme plays an important role in many diseases of altered cellular metabolism including obesity, insulin resistance, and dyslipidemia. Although SCD1 activity is highest in lipogenic tissues such as the liver and adipose tissue, it is also present at lower levels in most tissues. To better understand the role of SCD1 in liver metabolism it is necessary to explore SCD1 deficiency in a more focused, tissue-specific manner. This commentary focuses on 2 recent studies published in the JCI that address this question using antisense oligonucleotide inhibition of SCD1. First, Jiang et al. have previously reported that long-term inhibition of SCD1 prevents the development of high-fat diet-induced obesity and hepatic steatosis. Second, Gutiérrez-Juárez et al. show in this issue that short-term inhibition of hepatic SCD1 is sufficient to prevent diet-induced hepatic insulin resistance, signifying an important role of hepatic SCD1 in liver insulin sensitivity (see related article beginning on page 1686).

Nonstandard abbreviations used: ACC, acetyl-CoA carboxylase; AMPK, AMP kinase; ASO, antisense oligonucleotide; MUFA, monounsaturated fatty acid; PTP1B, protein tyrosine phosphatase 1B; SCD, stearoylCoA desaturase; TG, triglyceride.

Conflict of interest: The authors have declared that no conflict of interest exists.

Citation for this article: J. Clin. Invest. 116:1478-1481 (2006). doi:10.1172/JCI28774.
Stearoyl-CoA desaturase (SCD) is the central lipogenic enzyme catalyzing in vivo reactions in the synthesis of monounsaturated fatty acids (MUFAs), particularly oleate (C18:1n-9) and palmitoleate (C16:1n-7), which are the major MUFAs of membrane phospholipids, triglycerides (TGs), wax esters, and cholesteryl esters. Recent studies of SCD1 have yielded many new insights into the biology of lipid metabolism and have demonstrated that mice with a global deletion of $S c d 1$ (Scd1 1/- mice) are resistant to high-fat dietinduced obesity and glucose intolerance (1). Scd1 $1^{-/-}$mice also have increased hepatic fatty acid oxidation and decreased lipogenic gene expression (1). Furthermore, Scd1 $1 /-$ mice exhibit increased thermogenesis and insulin signaling in skeletal muscle and brown adipose tissue (2-4). Currently the mechanisms leading to the phenotypes due to SCD deficiency or overexpression in different tissues are not understood; yet the findings so far reveal that SCD1 is an important metabolic control point in lipid metabolism and is a promising drug target for the treatment of the metabolic syndrome.

\section{Can obesity and symptoms of the metabolic syndrome be alleviated by inhibition of SCD1?}

In vivo antisense oligonucleotide (ASO) reduction of target genes is a powerful tool for 